\title{
Review of current thermal imaging temperature calibration and evaluation facilities, practices and procedures, across EURAMET (European Association of National Metrology Institutes)
}

\author{
By H. McEvoy ${ }^{\star}$, R. Simpson* and G. Machin* ${ }^{\dagger}$
}

\author{
${ }^{*}$ National Physical Laboratory, Hampton Road, Teddington, Middlesex, TW11 OLW, UK, \\ helen.mcevoy@npl.co.uk \\ ${ }^{\dagger}$ Visiting Professor Universities of Glamorgan and Valladolid
}

\begin{abstract}
This paper provides an overview of, and the key findings from, a survey of the thermal imager 'temperature measurement' calibration and evaluation facilities, practices and procedures implemented within the EURAMET region (European Association of National Metrology Institutes, www.euramet.org).
\end{abstract}

\section{Introduction}

Thermal imaging (infrared, IR) cameras were developed towards the end of the Second World War for the military objectives of target recognition and identification. Military thermal imaging continued to develop over the following decades. Commercial thermal imagers were not commonly available until the mid-1960s, with the first systems being typically specialist, un-practical and expensive, meaning that uptake of the technology was low. The development of second and in particular third generation thermal imagers (i.e. those with uncooled staring focal plane arrays, available from the mid-1980s onwards) moved the thermal imaging sector towards the development of more practical devices resulting in a growth in commercial (non-military) uptake. Today, there is a wide availability of practical, versatile instruments, with introductory thermal imagers available for approximately $€ 1000$ (2012), leading to wide ranging and increasing usage. Indeed, recent market reports have stated:

- Infrared cameras are the fastest growing and most dynamic segment of the temperature sensing market, with currently $9.4 \%$ growth per annum [1];

- Infrared technology is currently the second largest segment (in temperature sensing technology), but is predicted to hold the prime position worldwide by 2014. A significant contributor to this is the growth of thermal imaging [1];

- In the next 5 years, the annual number of un-cooled, small array imagers sold is predicted to more than treble, and unit costs anticipated to more than halve [2];

This rapid growth in uptake points towards a potentially significantly disruptive "instrumentation of choice" change, where thermal imagers either open new measurement fields, or displace current measurement instruments. Taking the latter case, the non-contact temperature measurement "instrument of choice" has traditionally been the single spot infrared thermometer - an established historic technology designed for non-contact temperature measurement. The move from the use of single spot thermometers to thermal imagers for quantitative (and traceable) temperature measurement has already been raised as a potential "cross-roads" for the future of infrared technology [3].

In order to identify the current capability level in terms of temperature calibration and evaluation facilities, practices and procedures for thermal imagers within the EURAMET ${ }^{1}$ area (institutes charged with providing the measurement support framework) a survey was instigated under the auspices of the Consultative Committee of Thermometry $(\mathrm{CCT})^{2}$. This was done to identify what directed/structured foundation metrology work was required in the National Measurement Institutes (NMIs) to support this growth in quantitative thermal imager usage. This is a necessity since the calibration and evaluation of thermal imagers, although similar, has different requirements to single spot radiation thermometers. This is in part due to the fact that thermal imagers are array devices and not single detector devices, and in part because of the different design philosophies of the two devices. In general single spot radiation thermometers were specifically designed as temperature measurement devices, whereas, historically at least, thermal imagers were for target recognition and identification with little emphasis being given to reliable temperature measurement. However, it must be said that this latter aspect has been addressed in many modern thermal imagers.

The paper introduces the survey, describes the results and discusses the measurement capabilities required to support this growth in quantitative thermal imaging in the next few years.

\footnotetext{
${ }^{1}$ European Association of National Metrology Institutes: http://www.euramet.org/

2 The CCT is a consultative committee of the CIPM, the International Committee of Weights and Measures
} 


\section{Thermal imaging temperature calibration and evaluation facilities, practices and procedures}

A survey was sent to the leading experts in the field of non-contact (IR) temperature metrology within each National Metrology Institute (NMI) in the EURAMET region (37 NMIs). Seventeen responses were received; of these 2 institutes were not currently actively assessing thermal imagers. The survey content and overview of the responses from the remaining 15 Institutes are given below.

\subsection{Survey}

Information was requested on the following areas of evaluation for thermal imagers:

- Calibration methodology: calibration sources used for thermal imager calibration; available calibration temperature range; recommended temperature calibration points (if any); which portion(s) of the image are considered for the calibration; calibration distance or range of distances offered; documentary standards or procedures followed;

- $\quad$ Size-of-source effect (SSE): whether this is measured, and, if so, how it is measured and how the effect is propagated to temperatures other than that (or those) at which the assessment takes place; whether investigation of the SSE is included as standard within the assessment of thermal imagers;

- Distance effect (i.e. the effect of calibration distance on the imager reading): whether this is investigated, and whether the calibration is carried out at one set distance, or various;

- Field uniformity: whether this is investigated, and, if so, how it is measured and reported; whether a component for non-uniformity is included in the calibration uncertainty and how this effect is propagated to temperatures other than that (or those) at which the assessment took place;

- Environmental effects: whether the effect of changing environmental conditions on the calibration result is investigated, and, if so, how it is reported; whether a component is included in the calibration uncertainty and how the effect is propagated to other calibration temperatures;

- Emissivity correction: whether the device is calibrated at emissivity settings other than $1^{*}$, and, if so, how; whether or not the ambient temperature correction is checked; ( ${ }^{*}$ a good blackbody calibration source would have an emissivity of very close to 1 );

- Other: any other testing, e.g. MRTD (minimum resolvable temperature difference), NETD (noise equivalent temperature difference), MDTD (minimum detectable temperature difference); whether or not calibration of thermal imagers is a routine calibration service; whether the calibration is accredited by a national accreditation body; the calibration measurement capability (CMC; i.e. the calibration uncertainty typically expected to be achieved when calibrating a near-ideal instrument) of the NMI.

\subsection{Findings}

The responses from the 15 NMls were collated and are described here.

\subsubsection{Calibration methodology}

Predominantly, the NMIs use blackbody calibration sources that would be the same as those used for calibrating single spot radiation thermometers. However, three laboratories have flat/ grey plate radiators and one has a temperature source with a collimator and MRTD simulator. Five laboratories (33\%) have access to a source with an aperture diameter of $150 \mathrm{~mm}$ or larger.

The minimum calibration temperature offered is $-80^{\circ} \mathrm{C}$ and the maximum is $3000{ }^{\circ} \mathrm{C}$, with the most common temperature range (i.e. broadly achievable by the majority of the respondents) being $-30^{\circ} \mathrm{C}$ to $1000^{\circ} \mathrm{C}$. Most commonly, the calibration is carried out at 5 temperatures over the operational range of the instrument, although some laboratories carry out measurements at more temperatures, and others are flexible (i.e. the number of points is according to the customer's requirements). The most common calibration distance (distance from the blackbody source aperture to the imager) is $0.5 \mathrm{~m}$, although some laboratories offer up to $1 \mathrm{~m}$ or further.

$60 \%$ of the respondents did not specify the number of pixels used for the calibration measurement. The other $40 \%$ use, in general, pixels within the central portion of the image, although the specifics vary, ranging from $10 \%$ of the imager field of view to $1 / 3$ of the pixels within the central area, with one laboratory using all the pixels within the image over a limited temperature range. Usually, the average results from the central portion of the image are reported in the calibration certificate.

Only two of the NMIs follow International Standard guidelines for the calibration of thermal imagers, namely OIML R 141: 2008 (E) [4]. A third NMI follows an internal calibration procedure which is primarily used for the calibration of spot radiation thermometers. 


\subsubsection{Size-of-source effect (SSE)}

The SSE (see for example [5]) describes the effect of changing source size on the temperature indicated by the imager. It is caused by imperfections in the optical components of the imager and also by cross-talk between pixels. $40 \%$ of the NMIs stated that they routinely measure the SSE of the imager, although a further 33\% will do it on request and one laboratory would do it for new devices only. The SSE is nearly always assessed using a source (either a blackbody or greybody) with a variable aperture, but two laboratories use a greybody with masks and a third uses a scanning method (scanning the imager across the source). Most of the NMls report the results in the form of a table or graph showing the imager indicated temperature versus source diameter. With regard to the uncertainty, mostly only a nominal value or a value over a limited source diameter range is included in the uncertainty budget; only one NMI provides a full range of uncertainties over all source sizes. About $42 \%$ of the laboratories who measure the SSE measure at one temperature only and then propagate the uncertainty to different calibration temperatures using a model for the effective operating wavelength of the imager. The remaining laboratories either measure at one temperature only and don't propagate, or measure the SSE at each, or a number, of the calibration temperatures.

\subsubsection{Distance effect}

$20 \%$ of those responding routinely measure the distance effect, although a further $40 \%$ could do it at the request of the customer. Generally (67\% of respondents), the calibration is carried out at a set distance, with this distance stated in the calibration certificate. One NMI sets the distance so that the field-of-view of the imager is two times smaller than the diameter of the source, or so that the imager is at the minimum focal distance. Another NMI investigates the effect of defocusing or minor distance variation, while another investigates the effect over $\pm 20 \%$ of the calibration distance.

\subsubsection{Field uniformity}

$60 \%$ of the institutes routinely measure the imager field uniformity, with a further $13 \%$ measuring it on request. The uniformity is usually measured by positioning a blackbody source at a number of different points (specifically either 5 or 9 were stated) within the field-of-view (FOV) of the imager. However, a couple of institutes have a large aperture blackbody or a grey plate radiator whose temperature uniformity is very well known, and which will completely fill the FOV of the imager. All NMIs that measure the field uniformity report it by providing a table of measured values. The method for including it in the uncertainty budget varies; for example, the maximum observed difference is used, or the full range over the entire FOV (one NMI) or the variation over the central $25 \%$ or $33 \%$ of the image (most respondents). To propagate the uncertainty to the other calibration temperatures some laboratories utilise a model using the effective operating wavelength of the imager, whereas others measure the effect at all temperatures. Some NMIs only measure at one temperature and do not propagate the value.

\subsubsection{Environmental effects}

Very few institutes (13\%, 2 respondents) routinely perform any environmental tests, with another offering it on request. However, these checks are very limited: of those that specified details, one NMI changes the ambient temperature from $18{ }^{\circ} \mathrm{C}$ to $28{ }^{\circ} \mathrm{C}$, another NMl checks the influence of changing carbon dioxide and/ or water vapour in the line-of-sight between the imager and the calibration source. Other NMIs felt that it was sufficient simply to limit the amount of time the imager is in front of the calibration source, and one NMI only takes into account how the imager temperature reading varies with the natural variation in environmental conditions seen over the few minutes of the calibration measurement. Generally, if environmental checks are carried out, the results are reported, although one NMI would only report the results if the effect were significant. Only one NMI includes a component in the uncertainty budget for the effect. Other NMIs simply state the environmental conditions at the time of the calibration, although two would include the uncertainty if significant or if necessary.

\subsubsection{Emissivity correction}

$33 \%$ of the institutes calibrate the imager at different emissivity settings, with specific values given as $0.95,0.97$ and 0.99 . This is achieved using a blackbody or a greybody of a known emissivity. Only one NMI compares the change in the imager reading as the emissivity setting is varied with the theoretical model based on the effective operating wavelength of the imager and the ambient temperature. Only two respondents check that the imager ambient temperature correction is right.

\subsubsection{Other}

$33 \%$ of the institutes confirmed that they test other parameters related to imager performance. These included: short term stability over a 15 hour period (although this would not be carried out for every imager undergoing calibration); the temperature resolution; the drift between each internal calibration of the imager; the MRTD using a MRTD simulator 
with a temperature differential between $10 \mathrm{mK}$ to $10 \mathrm{~K}$; the MDTD (in accordance with ASTM standards if requested); testing according to OIML R 141 [4].

Out of the 15 laboratories, 6 laboratories (40\%) have a routine calibration service for thermal imagers, but only 4 ( $27 \%$ of the total) have an accredited calibration service, and this is primarily only if the imager is treated like a spot radiation thermometer. Over $50 \%$ of the laboratories either have no recognised Calibration Measurement Capability (CMC) for thermal imager calibration, or did not specify it. For those who did provide their CMCs, the values ranged from $0.2^{\circ} \mathrm{C}$ up to $7^{\circ} \mathrm{C}$ over the temperature range from $-40^{\circ} \mathrm{C}$ to $1500{ }^{\circ} \mathrm{C}$ depending on the laboratory.

\section{Discussion:}

The physical principles behind thermal imaging are essentially the same as for spot radiation thermometers, meaning that imagers are affected by the same issues and require similar calibration methods and equipment. However, there are also additional factors that need to be investigated, such as image non-uniformity and, depending on the application, MDTD, MRTD etc..

It appears from the results of the survey that the majority of the European NMIs who responded do not have a dedicated or special set-up for calibrating thermal imagers. Instead, they treat them like spot radiation thermometers and use the same facilities, with the calibration being carried out in terms of the temperature indicated by (generally) a number of pixels within the central portion of the image. This means that the imager calibrations are commonly being carried out using blackbody sources with relatively small aperture sizes. These have the advantage of having a high emissivity without the source being overly large. The disadvantage for imager calibrations is that the source only fills a relatively small part of the image. In order to assess the entire image it is necessary to carry out multiple measurements with the source positioned at different points within the image. Flat plate or greybody sources are available which offer larger source sizes, fill more of the image and provide easier temperature calibration and uniformity assessment of the whole image. However, the emissivity of these flat plate sources is lower, leading to larger temperature correction errors and more sensitivity to the effect of reflected thermal radiation from the surroundings. Further investigations and research are needed to determine whether it is sufficient to measure the imager non-uniformity by re-positioning the calibration source within the image, or whether a large area target with high emissivity and good temperature uniformity is required.

Evaluation of the SSE is routinely carried out by just under half the respondents, usually by using a blackbody or greybody source and variable aperture. Measurements of the SSE of imagers at NPL, looking the variation of the temperature indicated by the pixels within a central area within the image versus source size, have given results that are on a par with those expected from spot radiation thermometers. It means that (when viewing sources above ambient temperature) the indicated temperature increases as the source size increases. Some of the SSE will be as a result of imperfections in the optical components (as for spot radiation thermometers); another component is due to cross-talk between the pixels. The SSE has implications for the temperature accuracy of imagers, for example when viewing a target of a different size from that used in the calibration, or when measuring a surface that has significant temperature gradients. In order to improve temperature measurement with thermal imagers, it is necessary to know the magnitude of the SSE of the device. Since users might be carrying out measurements using the whole of the image, this would require a large area source so that the optical system of the imager can be filled or over-filled, and these are generally not available to the majority of the NMls surveyed.

The vast majority of European NMIs do not routinely carry out checks into the effect of changing distance from the source or changing environmental conditions. This could have implications for the results subsequently obtained with the imagers, since they are typically not used within the controlled environment of the laboratory and at a set distance from the target. Some investigations have shown that some imagers have poor compensation for changes in ambient temperatures [6,7]. Changes in an imager reading of the order of $0.5{ }^{\circ} \mathrm{C}$ have been observed at NPL following a relatively modest $\left(3^{\circ} \mathrm{C}\right)$ change in the laboratory temperature. In addition, a lot of imagers operate over the 3 to 5 micron wavelength range which contains a carbon dioxide absorption band. If this absorption band is not adequately filtered out, the imager reading will be influenced by varying levels of atmospheric carbon dioxide, either due to natural variation or as a result of changing path length between the imager and the target. In some extreme instances, this effect can be severe: for one imager tested at NPL, the sensitivity to $\mathrm{CO}_{2}$ was such that breathing in its line-of-sight completely obscured the image of the calibration source. It is important, therefore, that the effect of changing environmental conditions on the imager reading is assessed as fully as possible, to ensure that the results of the calibration are fit for the purpose for which the imager is subsequently to be used, within reasonable uncertainty limits.

The vast majority of respondents also do not routinely carry out any special additional measurements or checks such as the MDTD and MRTD, emissivity compensation algorithm etc. Also, interestingly, although international standards for calibrating and testing thermal imagers exist, in particular the OIML document [4], only two of the laboratories follow its recommendations. Only 4 have an accredited (i.e. independently assessed) calibration service, and this is primarily only if the imager is treated like a spot radiation thermometer.

Considering the components included in the calibration uncertainty for the imager for, for example, SSE, field non-uniformity etc., not all NMIs include all relevant factors in the uncertainty budget, meaning that the user is not always provided with the maximum information about the performance of his device. Also, since the magnitude, and hence the uncertainty, of these effects will often depend on the temperature of the target being measured, it is necessary to estimate the uncertainties for all the calibration temperatures; currently, not all of the NMIs are doing this. 


\section{Conclusions}

Thermal imagers have traditionally been used for qualitative assessments (for example for military target recognition). Increasingly, however, they are being used in a wide range of applications for quantitative temperature measurements, a field historically the domain of single spot radiation thermometers. For these applications there is a need to ensure that thermal imagers are correctly evaluated and calibrated in terms of temperature to reduce measurement errors and to ensure that users can have confidence in the results obtained.

The survey of calibration/evaluation sources and procedures within the EURAMET region reveals that common practices for thermal imager assessment are not currently in place, most laboratories utilising the calibration sources and procedures used for spot radiation thermometers. There exists some doubt and uncertainty as to the correct source, methodology, results reporting and uncertainty assessment to be used for thermal imagers. Furthermore, very few NMIs offer an accredited calibration service for thermal imagers. Several laboratories do, however, state that sections of the surveyed topics are current areas of research for the institute, e.g. the development of new procedures, sources etc. In addition, research is currently underway in a number of institutes into a variety of calibration/ evaluation parameters to fully understand their effect on quantitative temperature assessment.

With the rapidly increasing uptake and application of quantitative thermal imaging, it is essential that the metrological infrastructure be established to support this. This might involve the design and development of better quality large area calibration sources with a high and well-known emissivity and good temperature uniformity, both for temperature calibration and for a more rigorous assessment of the SSE and image non-uniformity. Environmental effects could also be better investigated, and this might require more widespread use of temperature controlled chambers, as well as some means to check the influence of atmospheric carbon dioxide and water vapour. The survey results also suggest that a common approach be agreed to the calibration and assessment of thermal imagers. In particular, further work is required to understand and quantify uncertainty sources (including field uniformity, SSE etc.) over the entire calibration temperature range so that users are provided with the maximum amount of information for optimum use of the imager.

Another, but important point, is that NMIs need to consider that many users might be new to non-contact temperature measurement, and they need to be educated to ensure that measurement results obtained 'in the field' are correctly interpreted and factors which influence the temperature reading of the imager are fully understood and taken into account.

\section{REFERENCES}

[1] "World Temperature Sensor and Transmitter Market", Frost and Sullivan, 2009.

[2] "Uncooled IR cameras \& Detectors for thermography and vision", Yole Development, 2010.

[3] Machin G., Simpson R., Broussely M., "Calibration and validation of thermal imagers", QIRT Journal, vol. 6, pp. 133-147, 2009.

[4] International Recommendation OIML R 141 Edition 2008 (E) 'Procedure for calibration and verification of the main characteristics of thermographic instruments', International Organization of Legal Metrology.

[5] Lowe, D., Battuello, M., Machin, G., Girard, F., "A comparison of size of source effect measurements of radiation thermometers between IMGC and NPL", Proceedings of Temperature its Measurement and Control in Science and Industry, vol. 7, ed. Ripple D., AIP Conference Proceedings, Chicago, pp. 625-630, 2003.

[6] Vendt R. et al, "Effects of Environmental Conditions on the Performance of Thermal Imagers" Int J Thermophysics, vol. 32, numbers 1-2, pp. 248-257, 2011.

[7] Pusnik, I., 'Reliability of Thermal Imaging in Calculation of Energy Efficiency in Buildings' Acta Metrologica Sinica, 29, pp 194 to198, 2008 